\title{
Aprender ciencias con visitas escolares: Características y dificultades desde la perspectiva del profesorado
}

\author{
Felipe Contreras-Baeza \\ Universidad Católica Silva Henríquez. Chile. https://orcid.org/0000-0001-6293-0797 \\ Correo para la correspondencia: felipe.anka@gmail.com
}

Artículo de Estudios y tendencias. Recibido: 09/11/2021. Aceptado: 25/11/2021. Publicación avanzada: 10/12/2021. Publicación: 03/01/2022.

\begin{abstract}
Resumen
Los desafíos de la educación científica actual requieren de una alfabetización científica que invite a las personas a posicionarse ante el mundo con una mirada crítica, informada y curiosa que les permita participar e involucrase en las diversas temáticas que competen e impactan a la sociedad. Para lograr este fin la escuela ha tenido que abrir sus puertas y reconocer el valor de otros contextos educativos que abordan temáticas científicas generando puentes entre ambos escenarios. De esta manera, las visitas escolares se sitúan como recursos y experiencias idóneas para favorecer una adecuada alfabetización científica pues permiten generar aprendizajes diversos e integrales. No obstante, el desconocimiento y la formación del profesorado mejorable sobre esta temática obstaculizan y dificultan la construcción de experiencias de aprendizaje entre escuela y museos y centros de ciencia que promuevan esta alfabetización.

\section{Palabras clave}

Alfabetización científica, Visitas escolares, Museos, Centros de ciencia.

\section{Referencia recomendada}

Contreras-Baeza, F. (2022). Aprender ciencias con visitas escolares: Características y dificultades desde la perspectiva del profesorado. REIRE Revista d'Innovació i Recerca en Educació, 15(1), 1-15. https://doi.org/10.1344/reire.37189
\end{abstract}

(c) 2022 El autor. Este artículo es de acceso abierto sujeto a la licencia Reconocimiento 4.0 Internacional de Creative Commons, la cual permite utilizar, distribuir y reproducir por cualquier medio sin restricciones siempre que se cite adecuadamente la obra original. Para ver una copia de esta licencia, visite https://creativecommons.org/licenses/by/4.0/ cc (i) 


\section{Títol (català)}

Aprendre ciències amb visites escolars: característiques i dificultats des de la perspectiva del professorat

\section{Resum}

Els desafiaments de l'educació científica actual requereixen una alfabetització científica que convidi les persones a posicionar-se davant el món amb una mirada crítica, informada i curiosa que els permeti participar i involucrar-se en les diverses temàtiques que incumbeixen i impacten a la societat. Per aconseguir-ho, l'escola ha hagut d'obrir les portes, reconèixer el valor d'altres contextos educatius que tracten temàtiques científiques, i generar ponts entre tots dos escenaris. D'aquesta manera les visites escolars se situen com a experiències i recursos idonis per afavorir una adequada alfabetització científica, perquè permeten generar aprenentatges diversos $i$ integrals. Tot $\mathrm{i}$ això, el desconeixement i la feble formació del professorat en aquesta temàtica dificulten la construcció d'experiències d'aprenentatge entre escola, museus i centres de ciència que promoguin aquesta alfabetització.

\section{Paraules clau}

Alfabetització científica, Visites escolars, Museus, Centres de ciència.

\section{Title (English)}

Learning science with school visits: Characteristics and difficulties from the teaching staff perspective

\section{Abstract}

The challenges of science education today require a scientific literacy that invites people to position themselves in relation to the world: with a critical, informed and curious look which allows them to participate and get involved in the various issues that concern and impact society. To achieve this goal, the school has had to open its doors and recognize the value of other educational contexts that address scientific topics, generating bridges between both scenarios. In this way, school visits are ideal resources and experiences to promote an adequate scientific literacy, since they generate diverse and integral learning. However, the lack of knowledge and the teacher training that can be improved on this subject hinder and make it difficult to build learning experiences between schools and museums and science centers that promote this literacy.

\section{Keywords}

Scientific literacy, School visits, Museums, Science centers. 


\section{Introducción}

Aprender ciencias hoy es una actividad necesaria y relevante en la educación de todo el mundo porque hay que tomar decisiones importantes y participar de la sociedad con conocimientos suficientes para comprender, debatir y consensuar temas relacionados con la ciencia, la tecnología, la sociedad y el medio ambiente. Ello nos invita a mirar el mundo con ojos científicos, lo cual representa para Furman et al. (2019) ese deseo inherente y natural de explorar y conocer el mundo para construir y desarrollar herramientas que nos permitan comprender cómo funcionan las cosas y poder pensar por nosotros mismos. De la misma forma, también se trata de que esa satisfacción y placer que se gana al comprender el mundo logre alimentar la llama de la curiosidad y la mantenga viva.

En ese sentido, mirar el mundo con ojos científicos significa desarrollar un proceso de alfabetización científica (Acevedo, 2004; Bybee \& McCrae, 2011; Garzón \& Martínez, 2017; OCDE, 2008; Soobard \& Rannikmäe, 2011) que consiste en la adquisición de conocimientos científicos que nos permitan cuestionar nuestro entorno, hacernos preguntas sobre él y tomar decisiones basadas en evidencia, entre otras cosas. Actualmente, la alfabetización científica no solo apunta a generar mayor número de profesiones científicas, sino que también aboga por un acercamiento de la ciencia hacia la población en general, bajo el lema de ciencia para todos o ciencia para la ciudadanía, donde hombres y mujeres, grandes y pequeños podamos habitar el mundo desde una perspectiva científica. Así lo confirman Couso et al. (2020) cuando mencionan que aprender ciencias en la actualidad se enfoca en formar ciudadanos críticos, "personas que, en un mundo inundado por la demagogia y las noticias falsas, sean capaces de pensar por sí mismas y elegir con criterio y libertad" (p. 7).

La educación científica tiene como fin último desarrollar este proceso de alfabetización. Por décadas ha sido la escuela la principal responsable de llevar a cabo esta misión. Sin embargo, los últimos acontecimientos relacionados con la pandemia de COVID-19 dan señales de la poca formación y preparación de la población en temas científicos (UNESCO, 2020). Así lo confirman diversos estudios internacionales sobre qué tan preparadas se sienten las personas sobre temas científicos y sus percepciones sobre ello (OCDE, 2019). En este sentido, parece ser que la educación formal, la escuela, no está cumpliendo del todo con este objetivo alfabetizador.

Cuando pensamos en la educación científica, se suele pensar únicamente en contextos formales de educación como escuelas, centros de formación y universidades. No obstante, existen además otros escenarios que, teniendo una intención educativa, también apuntan al desarrollo de la alfabetización científica: son los espacios de educación no formal de las ciencias, o también llamados museos y centros de ciencias, como museos interactivos, acuarios, planetarios y centros astronómicos, zoológicos, y otros. Estos centros tienen la cualidad de generar experiencias de aprendizaje diversas e integrales que permiten desarrollar conocimientos pertenecientes a la dimensión conceptual, procedimental o actitudinal que facilitan, a su vez, consolidar lo que algunos llaman la competencia científica (OCDE, 2019; Vera, 2012).

En este marco, para fortalecer y promover una adecuada alfabetización científica en las escuelas - generando experiencias de aprendizaje integrales- parece adecuado vincular y complementar el aprendizaje entre educación formal y educación no formal, entre escuela y museos y centros de ciencias. Al respecto, serían las actividades llamadas visitas escolares las instancias idóneas para ello.

No obstante, existen ciertas dificultades desde el punto de vista del profesorado para llevar a cabo este tipo de actividades de manera exitosa: algunas de ellas se deben a la formación mejorable que tienen sobre cómo vincular a ambos espacios (escuela y centros de ciencia), y otras a obstáculos administrativos. 
A continuación, se expone una breve revisión teórica sobre qué características tienen los aprendizajes generados en entornos de educación no formal, junto con cuáles debieran ser los componentes clave de una visita escolar. También quisiéramos compartir algunos hallazgos encontrados sobre dificultades del profesorado con la intención de aportar en conocimiento y experiencia al profesorado interesado en esta área y, sobre todo, a aquellos que desean salir del aula para fortalecer y ampliar los aprendizajes de la escuela.

\section{Aprender ciencias en los museos y centros de ciencia}

Gran parte del aprendizaje de las ciencias - a menudo no reconocido- se lleva a cabo fuera de la escuela, en contextos no formales e informales de educación (Touriñán, 1996; Trilla, 1993). En la educación formal, la enseñanza de las ciencias tiene como principal objetivo la alfabetización científica del estudiantado. Para lograr este propósito la OCDE (Werquin, 2010), la UNESCO (2000) y otros grandes organismos invitan a diseñar estrategias didácticas variadas, tanto en ambientes escolares como extraescolares, y es aquí donde los centros de educación no formal de las ciencias aparecen como un complemento ideal para su enseñanza y aprendizaje (Cuesta et al., 2000).

De todos estos escenarios, Linda Ramey-Gassert (1997) menciona que los museos y centros de ciencia tienen un potencial particular para favorecer el aprendizaje de la ciencia que la escuela no es capaz de desarrollar. Sobre este punto, la autora menciona que

Una distinción importante entre experiencias científicas dentro y fuera de la escuela es que el aprendizaje en un museo depende menos de símbolos verbales o escritos para la comunicación, permitiendo así a los alumnos interactuar con los objetos del mundo real sin el aprendizaje adicional de nuevos o confusos términos. Enseñar de la forma tradicional significa, en gran medida, el uso de símbolos en la lectura y matemática y sobre todo en la comunicación oral. En contraste, los entornos científicos informales ofrecen a los estudiantes experiencias directas que no son verbales, como objetos y exhibiciones visuales, en lugar de un discurso para transmitir información. (p. 434-435)

Ello hace que las experiencias en estos espacios requieran de una participación más activa del visitante como ver, tocar, oler, sentir o escuchar, entre otras acciones. Junto a esto, la autora agrega que los centros usan temas relacionados con la vida diaria, permiten la manipulación de elementos y objetos reales en contextos de interacción social, ofrecen la posibilidad de la libre elección con módulos interactivos, y otorgan un momento divertido. Dierking et al. (2003) destacan sobre todo el éxito de estos espacios para crear ambientes que facilitan la interacción social y el aprendizaje colaborativo, lo cual fomenta e invita a concebir una ciencia colaborativa.

Los museos y centros de ciencia entregan un amplio abanico de oportunidades y formas de aprendizaje. Es por esta razón que es complejo evaluar y medir los aprendizajes que ocurren dentro de estos espacios. Y es que aprender ciencia en estos contextos tiene características especiales porque es un proceso espontáneo, individual y a la vez social, que depende además del bagaje de conocimiento, ideas y experiencias previas y los intereses de las personas (Díaz y Hernández, 1999). Todo ello genera que las relaciones que establecen los visitantes entre sus propias ideas y la experiencia en el centro sean también distintas para cada persona, lo cual favorece la construcción de aprendizajes diversos (Hooper-Greenhill, 1995). Para Pérez et al. (1998), el grado de comprensión conceptual que se alcanza en la visita depende mucho de la edad, los conocimientos previos, la preparación de la visita y la participación del estudiantado.

Estos aprendizajes diversos se dividen en tres dimensiones: conceptual/cognitivo, procedimental/habilidades y afectivo/valórico. En el área cognitiva, el aprendizaje de las ciencias incluye "el conocimiento de hechos, fenómenos, principios y teorías, así como la aplicación de dichos conocimientos a las situaciones cotidianas" 
(Morentin, 2010, p. 24); lo cual favorece la dimensión conceptual del aprendizaje. Sobre esto mismo, la autora presenta la distinción entre el qué, el cómo y el por qué que plantea Wellington (1990). Según este autor, los museos y centros de ciencia tienden a responder únicamente el qué de los fenómenos y raramente se detienen a responder el cómo ocurren y por qué ocurren.

Wellington (1990) también comenta que son las dimensiones procedimentales y actitudinales las que se ven más favorecidas con las visitas a museos y centros de ciencia. Sobre el desarrollo de la dimensión procedimental, Pérez et al. (1998) comentan que estos espacios contribuyen de forma positiva al desarrollo de habilidades motrices y destrezas manuales y la coordinación óculo-manual, entre otras. Cigrik y Ozkan (2015), hablando específicamente de las habilidades de pensamiento científico, afirman que "el propósito principal de los centros de ciencias es proporcionar habilidades científicas y permitir que las personas alcancen el conocimiento en lugar de presentar solo la información" (p. 2). Los autores clasifican estas habilidades en dos grupos: las habilidades básicas como observación, exploración, registro, medición, clasificación, relación numérica y espacial, entre otras; y las habilidades de procesos integrados como formulación de preguntas e hipótesis, interpretación y análisis de datos, experimentación, entre otras. Al respecto, Griffin (1998) también afirma que los museos y centros de ciencia favorecen el aprendizaje de habilidades científicas, conocimientos que suelen quedar de lado en las actividades escolares por darle mayor énfasis a la dimensión conceptual.

Es quizás la dimensión afectiva/valórica de la ciencia la que se ve más estimulada gracias al aporte de estos centros, pues pueden provocar emoción, curiosidad y sorpresa, factores que juegan un rol clave en el aprendizaje (Pedretti, 2004). La dimensión afectiva o valórica comprende "el desarrollo del interés, el entusiasmo, el deseo de aprender, la creatividad, la apertura hacia los otros y hacia el ambiente... e incluso el deseo de llegar a ser científico" (Morentin, 2010, p. 24). Junto a ello, Griffin (1998) también menciona que las experiencias en estos espacios refuerzan una buena autoestima en el estudiantado y aumenta la motivación intrínseca para con la asignatura y el saber científico.

Con todo esto, es importante considerar que estos aprendizajes no siempre ocurren en el mismo centro o en el mismo instante. Wellington (1990) afirma que el aprendizaje de la ciencia puede aflorar semanas, meses e incluso años más tarde y, por lo tanto, la contribución de estos centros puede no ser necesariamente inmediata. Rennie (2014) lo expresa en una frase que refleja muy bien esta idea: "El aprendizaje que ocurre hoy depende del que se dio ayer y es la base del aprendizaje que ocurrirá mañana" (p.130), lo que lleva a pensar que el aprendizaje conlleva procesos y etapas que se dan en el tiempo, en el corto y largo plazo, que es un proceso de construcción y unión entre estos aprendizajes.

De esta manera, los aprendizajes conseguidos en un museo y centro de ciencia serían más amplios que un contenido conceptual específico, ya que también es posible desarrollar habilidades de pensamiento científico como la predicción y formulación de preguntas y actitudes como la confianza en sí mismo y la socialización de ideas. Es interesante pensar que estos aprendizajes se podrían conseguir visitando alguno de estos espacios, además de que estos pueden ser la base o consolidación para otros aprendizajes.

\section{La visita escolar: una oportunidad para aprender}

Por visita escolar se entiende toda experiencia didáctica que se realiza fuera del espacio escolar, en otro lugar, con el propósito de aprender. De esta manera, integramos espacios de educación no formal, que tienen una misión explícita con lo educativo, con aquellos espacios - los de educación informal-, que no declaran ni tienen objetivos definidos, pero donde igualmente ocurren aprendizajes, como una plaza, un parque, la calle, el bus, un supermercado u otro sitio. En la literatura es posible encontrar otros conceptos que hacen alusión a la misma 
idea: visita pedagógica, salida extraescolar, excursión, laboratorio al aire libre, viaje de campo, salida de campo u otras, que Rennie (2014) nos invita a considerar como sinónimos con una cierta flexibilidad. En este caso, se ha elegido el concepto visita escolar porque pensamos que en estas actividades es la escuela la que visita estos espacios.

Este tipo de actividad se caracteriza por utilizar otro espacio, que no es la escuela, generalmente para desarrollar, complementar o profundizar una cierta temática. Salir de la escuela en una actividad escolar significa que la escuela se está trasladando a otro espacio que generalmente también es educativo, aunque con ciertas diferencias. Estas diferencias son las que permiten que los aprendizajes conseguidos en un lugar u otro sean de distinta naturaleza, pero que se acompañan y complementan de buena manera. Sin embargo, existe un desconocimiento sobre cómo vincular y unir estos espacios educativos, y como consecuencia la experiencia y la visita escolar quedan aisladas del resto de procesos de aprendizajes previos y posteriores a los que se desarrollaron en el aula.

La investigación sobre visitas escolares lleva realizándose unos 30 años. Diversos autores han manifestado y reconocido el valor del aprendizaje de las ciencias en estas actividades y, con los años, han logrado consensuar ciertos componentes para que la visita escolar no quede aislada, sino que sea parte integrante del proceso de aprendizaje que se trabaja en la escuela.

La literatura señala que para que una visita escolar sea significativa debe estar construida considerando tres grandes componentes: presencia en la programación curricular, planificación respetando tres momentos -antes, durante y después - y utilización de estrategias didácticas que respondan al contexto en que se están usando. Veámoslo a continuación con más detalle en los siguientes apartados.

\subsection{Presencia en la programación curricular}

El valor del aprendizaje que sucede fuera de la escuela está aumentando cada vez más de tal manera que organismos internacionales como la UNESCO y la OCDE (Werquin, 2010) sugieren el uso de experiencias fuera del aula como recurso y complemento para la educación formal. En esa línea, para que el aprendizaje formal se vea favorecido por experiencias en entornos no formales es necesario que se reconozca el uso de este espacio como un entorno de aprendizaje dentro de la programación curricular. Incluir la visita escolar en la programación de la asignatura de manera explícita significa darle una presencia, un valor y un papel importante en el proceso de aprendizaje de los y las estudiantes. Dewitt y Hohenstein (2010) mencionan que incorporar la visita escolar en una o varias unidades didácticas contribuye a generar esa complementariedad entre ambos contextos, lo cual implica que el profesorado tenga que informarse sobre las actividades que puedan ofrecer los museos y centros de ciencia para conectar los objetivos de la visita con los de las actividades en aula.

\subsection{Planificación en tres momentos}

Darle una presencia a la visita escolar en la programación curricular significa que esta debe contar con una estructura y planificación. El profesorado debe preparar la actividad vinculando los objetivos que se están trabajando en el aula con aquellos que se trabajarán en el centro de ciencia. Para que dicha vinculación sea efectiva y la experiencia sea significativa se debe diseñar la visita sobre la base de tres grandes momentos: antes, durante y después de la visita (Griffin, 1998; Guisasola et al., 2005; Guisasola y Morentin, 2007, 2010; Morentin y Guisasola, 2013, 2014; NRC, 2010). 


\subsection{1. ¿Qué se hace antes de la visita?}

La experiencia que ofrece la visita escolar comienza antes de ir al centro de ciencia, antes de la visita misma, cuando se están diseñando los objetivos de la actividad, respondiendo al porqué y para qué de la visita. Es importante definir en la etapa previa a la visita cuál o cuáles son los objetivos, cuál va a ser su propósito, qué expectativas e intereses hay y otras preguntas que deberán ser trabajadas con el grupo curso; de esta manera, el estudiantado se implicará con la actividad (Morentin y Guisasola, 2014). Para Falk y Dierking (1997), cuando la visita escolar está conectada y vinculada con objetivos claros y conocidos por el estudiantado se favorece el proceso de aprendizaje en estos lugares, pues están involucrados con la actividad.

Así mismo, al consensuar cuáles son los objetivos de la visita también se definen qué temáticas se van a trabajar en el centro de ciencia, de esa manera se relacionan los contenidos del aula con los del museo. Hacer esta conexión es necesario para que la visita sea significativa, pues tiene que haber una relación entre los contenidos o conceptos que se extraen de una exhibición o montaje con los conocimientos que tiene el estudiante sobre este tema. Al respecto, Morentin (2010) sugiere que "al realizar las actividades previas será el momento de trabajar conceptos científicos concretos que se correspondan con las exhibiciones del centro de ciencia, para que una vez allí el alumnado pueda incorporar nuevas informaciones a su estructura conceptual" (p. 38). En concreto, en la etapa previa a la visita es necesario trabajar unos contenidos mínimos sobre cierto tema para que exista un cierto bagaje conceptual, una cierta familiarización con los conceptos que se van a trabajar en el centro de ciencia. Pero el trabajo en aula no se refiere solo a repasar o aprender nuevos conceptos, el trabajo previo incluye el uso de habilidades científicas como formulación de preguntas, observación, clasificación, análisis, generación de hipótesis y también, un trabajo de actitudes científicas como la curiosidad, creatividad, trabajo en equipo, entre otras.

De forma similar, Eshach (2007) comenta que es importante compartir el propósito de la visita y que el profesorado comparta sus propias expectativas para con la visita. Se debe acordar que la actividad sea considerada como una experiencia de aprendizaje. El mismo autor sugiere que visitemos las páginas webs de los centros, busquemos información e imágenes sobre ellos, presencia de videos en internet y cualquier dato que permita reducir la variable novedad, la cual podría influir negativamente en el aprendizaje, provocando episodios de ansiedad, incomodidad, inhibición del o la estudiante durante la visita, según mencionan Anderson y Lucas (1997). Es importante conocer qué material - como videos de presentación, cartas, lecturas o juegos - ofrecen los museos y centros de ciencia para trabajar en el aula y, a su vez, revisar el material de trabajo educativo que puedan ofrecer estos centros.

Por otro lado, Rennie y MacClafferty (1995) comentan que definir el propósito de la visita escolar es clave para hacer de la actividad una experiencia exitosa y efectiva. En este sentido, si la actividad surge como un reconocimiento, una recompensa o un premio al grupo curso, su desarrollo y resultado será muy distinto a si la visita se diseña como un recurso para complementar el aprendizaje formal.

\subsection{2. ¿Qué se hace durante la visita?}

La visita escolar como actividad que está incluida en la programación de la asignatura debe tener definidas la o las actividades que se realizarán en el centro de ciencia. En ese sentido, la visita escolar, al ser una actividad práctica, debe tener en cuenta ciertos aspectos para que sea una experiencia exitosa. Sobre dichos aspectos, Griffin (1998) comparte que "La visita debe propiciar experiencias que profundicen la comprensión de las ideas científicas" (p. 3). Los museos - a través de sus exhibiciones tradicionales, exhibiciones prácticas e interactivas, demostraciones, videos e imágenes - ofrecen experiencias que pueden relacionarse y vincularse con el 
conocimiento que ya se posee, y de esa manera se refuerzan los conceptos trabajados o también se les da sentido a los fenómenos cotidianos.

Griffin (1998) explica que la experiencia en el centro de ciencia debe acercar al estudiantado a cómo los científicos y científicas han generado el conocimiento: "La visita debe propiciar la realización de investigaciones mediante procesos científicos" (p.4), y para ello las actividades deben tener en cuenta la práctica de procedimientos científicos como formulación de preguntas e hipótesis, búsqueda de soluciones y respuestas, predicción y análisis de información entre otras.

Finalmente, y muy relacionado con la idea anterior, la autora menciona que "la visita debe propiciar la adquisición de habilidades de investigación científica” (p. 5). Estas habilidades podrían ser, por ejemplo, plantear preguntas, proponer formas de responderlas, examinar y evaluar, comparar, analizar, encontrar patrones de observación, aplicar ideas en situaciones nuevas, recopilar información, registrar observaciones, utilizar la evidencia de forma crítica, comunicar la información recopilada y comunicar los hallazgos.

En resumen, el profesorado deberá diseñar una visita escolar que permita vincular y unir los conceptos trabajados en el aula con aquellos que el centro de ciencia ofrece, a la vez que deberá diseñar actividades que favorezcan el desarrollo de habilidades científicas. Además, es importante que el profesorado tenga en cuenta que, pese a que la actividad es escolar, se está desarrollando en un espacio que tiene otra dinámica, por lo cual no puede realizar las mismas actividades que se hacen en la escuela, pues las características del centro hacen que el aprendizaje ocurra gracias a otros factores.

En concreto, para esta parte de la visita se reconoce la relevancia de diseñar actividades que permitan lograr los objetivos que se propusieron en la etapa previa a la visita, de manera que las actividades apunten a un acercamiento o profundización de temas científicos gracias a la exploración y procesos de trabajo científico en el centro.

\subsection{3. ¿Qué se hace después de la visita?}

El diseño de actividades para esta etapa debe considerar el trabajo y experiencias vividas en las dos etapas anteriores. Es común que el final de una visita escolar termine compartiendo las apreciaciones, sentimientos y vivencias dentro del centro; en otros casos se utilizan recursos didácticos tradicionales como guías, cuestionarios o informes para traducir algo de la experiencia. Repetir experiencias vividas en el museo, poner a dialogar las ideas previas de los estudiantes con las presentadas en el centro, diseñar trabajos prácticos, buscar información complementaria, formular preguntar y buscar sus respuestas y otras acciones que requieren un rol activo del estudiantado podrían ser buenas instancias para reforzar el aprendizaje de las ciencias (Morentin, 2010).

Las actividades propuestas en esta etapa deberían dirigirse a evaluar los objetivos propuestos para la visita, relacionar los conocimientos previos con los trabajados en el museo y valorar el aprendizaje utilizando estrategias que permitan ir concretando la experiencia de la visita escolar (Pedrinaci, 2012). Además, es importante volver reflexivamente al centro de ciencia e identificar qué procesos ocurrieron ahí en términos cognitivos, físicos, sociales y emocionales, como una forma de evaluar el impacto del centro de ciencia como recurso para el aprendizaje y como un lugar al cual se puede volver. 


\subsection{Utilización de estrategias didácticas}

Por último, el tercer componente para una buena visita es el uso de recursos y estrategias didácticas acordes al contexto educativo en el cual se van a utilizar. Existe un debate interesante sobre qué actividades realizar en el centro de ciencia durante la visita escolar. Es importante considerar que los espacios de educación no formal se caracterizan por ofrecer un entorno de aprendizaje más diverso y social, que ocurre en un escenario libre, movido por la curiosidad, el interés y la emoción (Rocha \& Bertelle, 2019). Considerando este contexto, el tipo de actividades debe responder a este espacio. Es común que el profesorado utilice estrategias similares a las que usa en el aula para trabajar en el museo, estrategias que representan acciones como completar fichas, hojas de campo, guías, cuestionarios o recursos similares (Griffin y Symington, 1997). Pero el uso de documentos puede restringir el proceso de aprendizaje porque la estructura de estos no se relaciona con la experiencia en los centros de ciencia, ya que no permiten que el estudiantado pueda reflexionar sobre lo que está aprendiendo y viviendo (Price y Hein, 1991). En cambio, Mortensen y Smart (2007) se inclinan por utilizar un recurso tipo hoja de trabajo en el que se tengan que resolver problemas relacionados con las exposiciones y que invite al estudiante a explorar y resolver estos problemas.

Eshash (2007) considera importante proporcionar al estudiantado una tarea específica que requiera su participación en donde tenga que observar, discutir, obtener información o formular preguntas, entre otras actividades. Estas tareas permitirían a los estudiantes ser conscientes de lo que está pasando a su alrededor; sin embargo, el tipo de actividades tiene que ser amigable pues no se puede saturar al alumnado con demasiadas tareas. Coincidiendo con Guisasola et al. (2005), "es necesario que ellos dispongan de tiempo para jugar y experimentar manualmente, así como con sus ideas, para plantearse sus propias preguntas y después buscar respuestas adecuadas" (p. 21).

\section{Percepciones del profesorado}

Diseñar visitas escolares que sirvan de puente entre la escuela y los museos y centros de ciencia para promover la alfabetización científica exige del profesorado un trabajo y un conocimiento adicional para el cual no siempre está formado. Se han dedicado diversos estudios a responder qué motivaciones, objetivos, expectativas y dificultades tiene el profesorado cuando decide realizar una visita escolar.

Una de las motivaciones principales para hacer una visita escolar a un museo o centro de ciencia es vincular los contenidos que se abordarán en ese espacio con aquellos que se están trabajando o se trabajarán en la escuela; en otras palabras, relacionar la visita con el currículo escolar. Así lo confirma el estudio de Anderson y Zhang (2003), en el cual el 60,6\% de profesores (de un total de 63 encuestados) respondió que hacía la visita siempre que fuera apropiada para el currículo escolar. De la misma forma, en el estudio de Viladot (2009), al preguntar sobre las motivaciones para hacer la visita, el $74 \%$ del profesorado (77 encuestados en total) respondió que lo hacía para ampliar los contenidos de la programación curricular. Kisiel (2005), por su parte, al entrevistar a 115 educadores sobre sus motivaciones, encontró que el $90 \%$ de ellos lo hacía para conectar la visita con el currículo y los contenidos del aula. De esta última investigación, llama la atención que un 39 \% del profesorado considera la visita como una oportunidad para entregar una experiencia rica y novedosa que de otra manera no podría tenerse. En otro estudio posterior, Viladot (2015) expone otras motivaciones, más sociales, refiriéndose a que muchas veces la visita escolar es la única oportunidad que tienen los estudiantes de salir y conocer un museo o centro de ciencia.

Las motivaciones del profesorado se complementan con los objetivos por los cuales realizan una visita escolar. La gran mayoría decide ir a un centro de ciencia para complementar y profundizar el aprendizaje de las ciencias y su 
relación con el currículo escolar. Para conocer estos objetivos, Guisasola y Morentin (2010) entrevistaron a 158 profesores de los cuales el 60 \% manifestó que lo hacía porque la visita "tiene como objetivo acercarse al mundo de la ciencia [...] en la visita tenemos como objetivo suscitar el interés por la ciencia" (p. 130), mientras que el $53 \%$ lo hacía para complementar las ideas trabajadas en el aula.

Por otro lado, Kisiel (2005) sostiene que, pese a que un gran grupo de profesores realiza la visita para profundizar y complementar el aprendizaje de la ciencia escolar, otro gran porcentaje lo realiza para ofrecer una experiencia de aprendizaje distinta a la que podrían vivir en la escuela. En ese sentido, plantea que las expectativas del profesorado para con los museos y centros de ciencias se resumen en las siguientes:

- los y las estudiantes pueden adquirir nuevo conocimiento relacionado con el currículo o no, como consecuencia de la visita;

- las experiencias realizadas por los mismos estudiantes son una contribución importante para su aprendizaje;

- la visita al museo podría brindar una perspectiva adicional o una conexión más significativa, que ayude a los estudiantes con alguna parte del currículo;

- la visita escolar puede brindar una experiencia completamente nueva para los estudiantes, y esta experiencia puede tener un impacto positivo en el desarrollo del alumno y el aprendizaje que suceda en el futuro; y

- la visita escolar puede despertar el interés por algún tema o concepto, y hacer que los estudiantes se sientan motivados por descubrir más.

Respecto a las dificultades que presenta el profesorado cuando quiere hacer una visita escolar, en la literatura se encuentran estudios que explican ciertos obstáculos que en este artículo se han clasificado en dificultades logísticas y didácticas. Las logísticas se refieren a aquellas acciones administrativas y de gestión que comporta una actividad de este tipo: transporte, distancia, autorizaciones, etc. Las didácticas, en cambio, se refieren a aquellas decisiones pedagógicas de la visita como qué actividades realizar, dónde, cuánto tiempo deben durar, qué preguntas hacer, qué material utilizar, cómo evaluar, etc.

Sobre los aspectos logísticos, Anderson y Zhang (2003) plantean algunas cuestiones que el profesorado tiene en consideración cuando plantea una visita escolar: el precio del transporte y el valor de la entrada al centro, la ubicación del espacio, la distancia con la escuela y la duración del viaje, los efectos de la visita en la organización interna de la escuela (cambio de horarios, asignaturas, rutinas), la seguridad del estudiantado en el centro, y la calidad de exposiciones/montajes y su relación con el currículo y aprendizaje formal. Estas y otras consideraciones podrían transformarse en obstáculos para la visita y dificultades para el profesorado. De forma similar, Michie (1998) comparte otras preocupaciones del profesorado como: la falta de apoyo de la administración de la escuela para efectuar la visita, la inflexibilidad del currículo, y la falta de oportunidades y conocimientos para elaborar recursos adecuados. Es esta falta de conocimientos y preparación la debilidad principal del profesorado para poder desarrollar visitas escolares efectivas y significativas.

Por ejemplo, en el estudio de Griffin y Symington (1997) no todo el profesorado tenía definidos los objetivos de la visita escolar, ni qué tipo de actividades se realizarían posteriormente. Sobre esto, Viladot (2009) considera que son pocos los profesores que desarrollan alguna actividad didáctica específica en el centro de ciencia. Guisasola y Morentin (2010) plantean que este hecho es contradictorio, pues el profesorado considera la visita escolar como una experiencia importante, pero la gran mayoría no diseña actividades específicas para trabajar en el aula después de la visita. Según estos autores, algunos de estos motivos serían la poca preparación y el poco tiempo de planificación que tienen los educadores. 
En resumen, algunos estudios (Anderson et al., 2003; Griffin, 1998, 2004; Griffin y Symington, 1997; Guisasola et al., 2005; Guisasola y Morentin, 2010; Kisiel, 2005; Morentin, 2010; Viladot, 2009) reafirman la poca implicación del profesorado con la visita pese a que la consideran valiosa y un aporte al desarrollo del aprendizaje formal de las ciencias. Pocas veces definen objetivos para la actividad y pocas veces la diseñan teniendo en cuenta los tres grandes momentos explicados en este artículo. De igual forma, pocas veces diseñan actividades posteriores a la visita para vincular los contenidos y aprendizajes adquiridos, haciendo que la experiencia quede aislada de la educación formal.

Griffin (2004) explica de forma muy clara por qué pasa esto. Según afirma, el profesorado no se implica y no se compromete con la visita escolar porque lucha con problemas de tiempo, logística, presiones económicas y administrativas, y todo esto obstaculiza su quehacer. Estas mismas dificultades son las que Anderson y Zhang (2003) plantean en su estudio, a las cuales hemos llamado dificultades logísticas. A esta situación se agrega la poca formación que recibe el profesorado para trabajar en contextos no formales e informales de educación en la formación inicial (Olson et al., 2001).

Finalmente, de acuerdo con Mortensen y Smart (2007), son muy pocos los estudios de diseño y elaboración de materiales y propuestas didácticas sobre visitas escolares que tengan en consideración todo lo que se ha revisado y que, a la vez, sean conocidos por el profesorado para planificar su enseñanza.

\section{Conclusiones}

El aprendizaje de las ciencias tiene la particularidad de que puede suceder en diversos escenarios y lugares, tanto en la escuela como fuera de ella, en museos y centros de ciencia y en la calle. Por este motivo parece adecuado generar vínculos y puentes entre estas experiencias educativas de manera explícita a través de las visitas escolares. En estas visitas, el aprendizaje logrado fortalece los conocimientos científicos trabajados en la escuela y a la vez favorece el desarrollo de habilidades de pensamiento y actitudes científicas.

Considerando lo anterior, tiene sentido que, hace ya un tiempo, diversas voces planteen el trabajo de complementariedad entre contextos educativos como una forma de enriquecer la ciencia escolar con aquello que se vive en el día a día. De esta manera sería adecuado el trabajo con visitas escolares desde los niveles iniciales de la educación escolar.

Pese a reconocer lo valioso de las experiencias fuera del aula, todavía es complejo realizar visitas escolares significativas y exitosas. Mucho de ello se debe a la mejorable formación del profesorado sobre este tema y, a la vez, a los obstáculos administrativos que surgen al generar una actividad de este tipo.

Para atender a la primera de las dificultades, se han revisado los hallazgos de la literatura sobre cómo preparar una adecuada visita escolar. Se ha mencionado que una buena visita escolar es aquella que tiene una presencia explícita en la programación curricular de la asignatura, de esta manera le damos voz, valor y relevancia dentro del abanico de actividades de la unidad. A la vez, se ha comentado que su preparación y diseño deben estar estructuradas en tres grandes partes que serían el momento previo a la visita, durante y después de la visita, entre las cuales se logre mantener una cierta lógica. Con ello se asegura de que las experiencias vividas en cada uno de los momentos se complementen entre sí con el propósito de generar nuevos aprendizajes y lograr los objetivos propuestos. Finalmente, se ha comentado la importancia de utilizar estrategias y recursos didácticos adecuados para el contexto de la visita escolar que vayan más allá de los utilizados en el aula con tal que puedan guiar el aprendizaje y no limitarlo. 
Finalmente, invitamos al profesorado a salir del aula y la escuela para generar experiencias de aprendizajes diversos y profundos. Hacemos un llamado para que se apropien de otros espacios y escenarios y se sirvan de ellos para generar una adecuada alfabetización científica. Consideramos que al hablar de visitas escolares es necesario conocer cómo estructurar estas actividades y conocer qué dificultades y percepciones tienen los colegas que han trabajado con ellas para aprender de las experiencias de otros y mejorar nuestra propia labor docente.

\section{Referencias}

Acevedo, J. A. (2004). Reflexiones sobre las finalidades de la enseñanza de las ciencias: educación científica. Eureka sobre Enseñanza y Divulgación de las Ciencias, 1, 3-16. https://doi.org/10.18260/1-2--10236

Anderson, D., y Lucas, K. (1997). The Effectiveness of Orienting Students to the Physical Features of a Science Museum Prior to Visitation. Research in Science Education, 27(4), 485-495. https://doi.org/10.1007/BF02461476

Anderson, D., Lucas, K. B., y Ginns, I. S. (2003). Theoretical perspectives on learning in an informal setting. Journal of Research in Science Teaching, 40(2), 177-199. https://doi.org/10.1002/tea.10071

Anderson, D., y Zhang, Z. (2003). Teacher Perceptions of Field-Trip Planning and Implementation. Visitor Studies Today, $\mathrm{VI}(3), 6-11$.

Bybee, R., y McCrae, B. (2011). Scientific literacy and student attitudes: Perspectives from pisa 2006 science. International Journal of Science Education, 33(1), 7-26. https://doi.org/10.1080/09500693.2010.518644

Cigrik, E., y Ozkan, M. (2015). The Investigation of the Effect of Visiting Science Center on Scientific Process Skills. 7th World Conference on Educational Sciences, 1312-1316. https://doi.org/10.1016/j.sbspro.2015.07.405

Couso, D., Jiménez-Liso, M. R., Refojo, C., y Sacristán, J. A. (2020). Enseñando ciencia con ciencia. Fundación Lilly, Fundación Española para la Ciencia y Tecnología (FECYT). https://www.fecyt.es/es/publicacion/ensenando-ciencia-con-ciencia

Cuesta, M., Díaz, M. P., Echeverria, I., Morentin, M., y Pérez, C. (2000). Los museos y centros de ciencia como ambientes de aprendizaje. Alambique, 26, 21-28.

Dewitt, J., y Hohenstein, J. (2010). Supporting Student Learning: A Comparison of Student Discussion in Museums and Classrooms. Visitors Studies, 13(1), 41-66. https://doi.org/10.1080/10645571003618758

Díaz, A., y Hernández, R. (1999). Constructivismo y aprendizaje significativo. En Estrategias docentes para un aprendizaje significativo (pp. 13-33). Mc Graw Hill.

Dierking, L., Luke, J., y Büchner, K. (2003). Science and technology centres - rich resources for free-choice learning in a knowledge-based society. International Journal of Technology Management, 25, 441-459. https://doi.org/10.1504/IJTM.2003.003112

Eshach, H. (2007). Bridging in-school and out-of-school learning: Formal, non-formal, and informal education. Journal of Science Education and Technology, 16(2), 171-190. https://doi.org/10.1007/s10956-006-90271 
Falk, J. H., y Dierking, L. D. (1997). School Field Trips: Assessing Their Long-Term Impact. Curator: The Museum Journal, 40(3), 211-218. https://doi.org/10.1111/j.2151-6952.1997.tb01304.x

Furman, M., Jarvis, D., Luzuriaga, M., y Podestá, M. E. (2019). Aprender a mirar el mundo con ojos científicos. En Aprender ciencias en el jardín de infantes (pp. 11-23). Aique.

Garzón, A., y Martínez, A. (2017). Reflexiones sobre la alfabetización científica en la educación infantil. Espiral. Cuadernos del Profesorado, 10, 28-39. https://doi.org/10.25115/ecp.v10i20.1010

Griffin, J. (1998). Learning science through practical experiences in museums. International Journal of Science Education, 20(6), 655-663. https://doi.org/10.1080/0950069980200604

Griffin, J. (2004). Research on Students and Museums: Looking More Closely at the Students in School Groups. Wiley InterScience, 59-70. https://doi.org/10.1002/sce.20018

Griffin, J., y Symington, D. (1997). Moving from Task-Oriented to Learning-Oriented Strategies on School Excursions to Museums. Science Education, 81, 763-779. https://doi.org/10.1002/(SICI)1098237X(199711)81:6\%3C763::AID-SCE11\%3E3.0.CO;2-O

Guisasola, J., Azcona, R., Etxaniz, M., Mujika, E., y Morentin, M. (2005). Diseño de estrategias centradas en el aprendizaje para las visitas escolares a los museos de ciencias. Revista Eureka sobre Enseñanza y Divulgación de las Ciencias, 2, 19-32.

Guisasola, J., y Morentin, M. (2007). ¿Qué papel juegan las visitas escolares a los museos de ciencias en la aprendizage de ciencia? Una revisión de las investigaciones. Enseñanza de las ciencias, 25(3), 401-414.

Guisasola, J., y Morentin, M. (2010). Concepciones del profesorado sobre visitas escolares a museos de Ciencias. Enseñanza de las ciencias: revista de investigación y experiencias didácticas, 28(1), 127-140.

Hooper-Greenhill, E. (1995). The educational role of the museum. Routledge.

Kisiel, J. (2005). Understanding elementary teacher motivations for science fieldtrips. Science Education, 89(6), 936-955. https://doi.org/10.1002/sce.20085

Michie, M. (1998). Factors influencing secondary science teachers to organise and conduct field trips. Australian Science Teacher's Journal, 44, 82-95.

http://members.ozemail.com.au/ mmichie/public_html/fieldtrip.html

Morentin, M. (2010). Los museos interactivos de ciencias como recurso didáctico en la formación inicial del profesorado de Educación Primaria [Tesis doctoral, Universidad del País Vasco]. http://hdl.handle.net/10810/6874

Morentin, M., \& Guisasola, J. (2013). Visitas escolares a centros de ciencias basadas en el aprendizaje. Alambique, $73,61-68$.

Morentin, M., \& Guisasola, J. (2014). La visita a un museo de ciencias en la formación inicial del profesorado de Educación Primaria. Revista Eureka sobre Enseñanza y Divulgación de las Ciencias, 11(3), 364-380. https://doi.org/10.25267/Rev_Eureka_ensen_divulg_cienc.2014.v11.i3.07 
Mortensen, M. F., y Smart, K. (2007). Free-choice worksheets increase students' exposure to curriculum during museum visits. Journal of Research in Science Teaching, 44(9), 1389-1414. https://doi.org/10.1002/tea.20206

NRC [National Research Council]. (2010). Surrounded by science: Learning science in informal environments. https://doi.org/10.17226/12614

OCDE. (2008). Informe PISA 2006 - Competencias científicas para el mundo del mañana. Revista Internacional del Trabajo, 125(4), 2-400.

OCDE. (2019). PISA 2018 Results (Volume I): What students know and can do. https://doi.org/10.1787/5f07c754en

Olson, J., Cox-Petersen, A., y McComas, W. (2001). The inclusión of informal environments in science teacher preparation. Journal of Science Teacher Education, 12(1-4), 155-173. https://doi.org/10.1023/A:1016715127697

Pedretti, E. G. (2004). Perspectives on learning through research on critical issues-based science center exhibitions. Science Education, 88, 34-47. https://doi.org/10.1002/sce.20019

Pedrinaci, E. (2012). Trabajo de campo y aprendizaje de las ciencias. Alambique: Didáctica de las ciencias experimentales, $71,81-90$.

Pérez, C., Díaz, M., Echeverría, I., Morentin, M., y Cuesta, M. (1998). Centros de Ciencia: espacios interactivos para el aprendizaje. Universidad del País Vasco.

Price, S., y Hein, G. (1991). More than a field trip: Science programs for elementary school groups at museums. International Journal of Science Education, 13, 505-519. https://doi.org/10.1080/0950069910130502

Ramey-Gassert, L. (1997). Learning Science beyond the Classroom. Elementary School Journal, 97(4), 433-451. https://doi.org/10.4135/9781473910850.n9

Rennie, L. (2014). Learning Science Outside of School. En N. Lederman \& S. Abell (Eds.), Handbook of Research on Science Education (Vol. II, pp. 120-144). Routledge.

Rennie, L., y Mcclafferty, T. (1995). Using Visits to Interactive Science and Technology Centers, Museums, Aquaxia and Zoos to Promote Learning in Science. Journal of Science Teacher Education, 6, 175-185. https://doi.org/10.1007/BF02614639

Rocha, A., y Bertelle, A. (2019). Educación no formal para el aprendizaje de las ciencias. La experiencia en el marco del Proyecto Difusión de la Ciencia en la Escuela. Anuario Digital de Investigación Educativa, 17, 1-10. http://revistas.bibdigital.uccor.edu.ar/index.php/adiv/article/view/3407

Soobard, R., y Rannikmäe, M. (2011). Assessing Student's Level of Scientific Literacy Using Interdisciplinary Scenarios. Science Education International, 22(2), 133-144. https://eric.ed.gov/?id=EJ941672

Touriñán, J. M. (1996). Análisis conceptual de los procesos educativos «formales», «no formales» e informales». Teoría de la Educación. Revista Interuniversitaria, 8, 55-79. https://doi.org/10.14201/3092

Trilla, J. (1993). La educación fuera de la escuela. Ámbitos no formales y educación social. Ariel. 
UNESCO. (2000). La ciencia para el siglo XXI. Un nuevo compromiso. https://unesdoc.unesco.org/ark:/48223/pf0000122938_spa

UNESCO. (2020). El estado de la ciencia. Principales indicadores de ciencia y tecnología Iberoamericanos. http://www.ricyt.org/wp-content/uploads/2021/02/ElEstadoDeLaCiencia_2020.pdf

Vera, J. (2012). Competencias científicas de docentes de educación superior tecnológica en México. Universidades, 52, 4-17. https://biblat.unam.mx/hevila/UniversidadesMexicoDF/2012/no52/1.pdf

Viladot, P. (2009). ¿Para qué vienen? Expectativas de los docentes en las visitas escolares al museo. Enseñanza de las ciencias: revista de investigación y experiencias didácticas, Extra, 520-524.

Viladot, P. (2015). Motivacions, expectatives i objectius del professorat en les visites als museus de ciència [Tesis doctoral, Universitat de Barcelona]. http://www.tdx.cat/handle/10803/396192

Wellington, J. (1990). Formal and informal learning in science: The role of the interactive science centres. Physics Education, 25(5), 247-252. https://doi.org/10.1088/0031-9120/25/5/307

Werquin, P. (2010). Recognising Non-Formal and Informal Learning. Outcomes, Policies and Practices. OECD. https://doi.org/10.1787/9789264063853-en 